\title{
National Evolutionary Synthesis Center
}

2024 W. Main St., Suite A200

Durham, NC 27705 USA

http://www.nescent.org

$919-668-4551$

\section{NESCent Data, Software and Publication Policy}

\section{Overview}

The open availability of data, software source code, methods, and results is good scientific practice and a key ingredient of synthetic research. In order to promote participation of the greater research community in synthetic evolutionary science, NESCent expects that all data and software created through NESCent-sponsored activities be made publicly available no later than one year after the conclusion of the NESCent award, or immediately upon publication of an associated article, whichever comes earlier. Exceptions may be granted at the discretion of the Director.

\section{NESCent-sponsored Data}

NESCent sponsored-data compilations are to be made accessible, with no restrictions on use and dissemination beyond attribution, through explicit application of a Creative Commons Zero waiver or its equivalent, and through deposition in a public data repository. The data should be adequately documented for validation and reuse, including appropriate attribution of its original source.

\section{NESCent-sponsored Software}

NESCent-sponsored software is to be made freely available to the research community by dissemination of the source code through a suitable public archive, explicitly licensed with a recognized Open Source License or a copyright waiver (e.g. $\mathrm{CC0})$. The documentation should at a minimum state the purpose, installation instructions, usage, copyright/license/waiver, and any necessary attribution of third-party software. NESCent encourages source code to be made available from the start of development, rather than delayed until the publication or after the conclusion of a project. Software developed by NESCent staff, including that in support of sponsored science projects, will be made publicly available from initiation of the project.

\section{NESCent-supported Publications}

To promote the widest possible access to and reuse of results from the research that it sponsors, NESCent encourages Open Access publication. NESCent will provide support for Article Processing Charges for qualifying publications. Limitations, eligibility, and caps for reimbursement are based on Duke University's implementation of the Compact for Open Access Publishing Equity. NESCent Postdoctoral Fellows are asked to provide matching funds from their research budgets when available. 


\section{Frequently Asked Questions}

- What constitutes NESCent-sponsored software or data?

- What qualifies as a public data repository?

- Why not use a license for data that requires attribution, share-alike or noncommercial restrictions?

- What is an "acceptable archive" for software?

- How do I choose an appropriate software license?

- How should copyright and license be stated for software?

- Can I embargo my data / software?

- Are any data or software exempt from this policy?

\section{What constitutes NESCent-sponsored software or data?}

Data compilations and software source code developed wholly or in part while a NESCent-sponsored scientist (Sabbatical Scholar, Graduate or Postdoctoral Fellow, Short-term Visitor, Fellow), as part of a NESCent-sponsored activity (such as a Working Group or Catalysis Meeting), or hosted on NESCent-controlled servers.

\section{What qualifies as a public data repository?}

Data in digital format must be downloadable without the requirement of a user account, the registration of personal information, a request for access, or restrictive terms of use. The repository should also be recognized by one or more peer-reviewed journals. NESCent encourages the use of the public data repository Dryad for NESCent-sponsored data when associated with a publication and when an appropriate datatype-specific repository is lacking.

\section{Why not use a license for data that requires attribution, share- alike or non-commercial restrictions?}

The complexities of copyright, ownership, licensing and attribution of scientific data are beyond the scope of this FAQ, and we point readers instead to the discussion of these issues at the Creative Commons. NESCent urges the use, for factual data, of a CC0 waiver because it does not falsely claim copyright that is not legally valid, nor does it impose attribution, share-alike and non-commercial restrictions that would limit the usefulness of that data for further synthetic research.

\section{What is an "acceptable archive" for software?}

NESCent's definition of an acceptable archive follows that of the PLOS. The code should be easy to locate and download without the requirement for a user account, the registration of personal information, a request for access, or restrictive terms of use. The repository should ideally have been in existence for over five years and host more than one thousand projects. Examples of such archives are: SourceForge, Bioinformatics.Org, Open Bioinformatics Foundation $(\mathrm{O}|\mathrm{B}| \mathrm{F})$, Google Code, BerliOS Developer, Savannah,

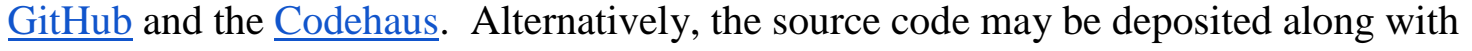
the data in a public repository (such as Dryad); however, this may require waiving copyright. 


\section{How do I choose an appropriate software license?}

Morin et al. (2012) (http://dx.doi.org/10.1371/journal.pcbi.1002598) give an accessible and comprehensive overview of how to choose among software licenses. NESCent's Informatics team will help in selecting the most suitable license, and will also help to remove other possible obstacles towards making data and software available, for instance by researching and resolving license incompatibilities, consulting or coordinating with collaborators, etc.

\section{How should copyright and license be stated for software?}

The copyright statement and license should at least be stated in a designated file, by convention called LICENSE.txt, in the root directory of the source code archive. We recommend to also include the copyright statement and a short form of the license in every source code file. This ensures that the information stays with the source code when it is redistributed, whether as a whole or in part.

\section{Can I embargo my data / software?}

NESCent policy does not allow a delay in release of the data relative to the time of article publication, even if such an embargo period is allowed by the journal and data archive.

\section{Are any data or software exempt from this policy?}

NESCent recognizes that exceptions to this policy may be needed in special cases. For instance, data or software may have pre-existing terms of use and dissemination that conflict with those set forth here. Requests for exemptions should be provided in writing to the Director. NESCent asks its sponsored scientists to consider obtaining agreements from external collaborators or institutions so as not to restrict sharing and free dissemination of the research outputs sponsored by the Center. 